Research Article

\title{
Singular Integral Equations of Convolution Type with Cosecant Kernels and Periodic Coefficients
}

\author{
Pingrun Li \\ School of Mathematical Sciences, Qufu Normal University, Qufu 273165, China \\ Correspondence should be addressed to Pingrun Li; lipingrun@163.com
}

Received 15 July 2017; Revised 30 August 2017; Accepted 7 September 2017; Published 9 October 2017

Academic Editor: Elena Benvenuti

Copyright (C) 2017 Pingrun Li. This is an open access article distributed under the Creative Commons Attribution License, which permits unrestricted use, distribution, and reproduction in any medium, provided the original work is properly cited.

\begin{abstract}
We study singular integral equations of convolution type with cosecant kernels and periodic coefficients in class $L^{2}[-\pi, \pi]$. Such equations are transformed into a discrete jump problem or a discrete system of linear algebraic equations by using discrete Fourier transform. The conditions of Noethericity and the explicit solutions are obtained by means of the theory of classical boundary value problem and of the Fourier analysis theory. This paper will be of great significance for the study of improving and developing complex analysis, integral equations, and boundary value problems.
\end{abstract}

\section{Introduction}

It is well-known that the boundary value problems for analytic functions have been widely used in many fields, such as engineering mechanics, physics, engineering technology, and fracture mechanics. Various types of boundary value problems for analytic functions and singular integral equations have been deeply studied and widely applied to practical problem (see [1-3]). In the theory of integral equations, the convolution type integral equations and singular integral equations are two important classes of equations, which had been studied by many mathematical workers and there were already rather complete theoretical systems (see $[4,5]$ ). These theories have been widely used in practical applications, such as engineering mechanics, fracture mechanics, and elastic mechanics (see $[6,7]$ ).

The main aim of this paper is to extend further the theory to singular integral equations of convolution type with periodic coefficients and cosecant kernel in class $L^{2}[-\pi, \pi]$ (see [8] for the definition of $L^{2}[-\pi, \pi]$ ). By using discrete Fourier transform, such equations are transformed into a discrete jump problem or a discrete system of equations. The explicit expressions of general solution and the conditions of solvability are obtained in class $L^{2}[-\pi, \pi]$. Therefore, this paper generalizes some results for [1-5].

\section{Definitions and Lemmas}

In this section we present some definitions and lemmas.

Definition 1. Suppose $\varphi=\left\{\varphi_{k}\right\}=\left\{\ldots, \varphi_{-1}, \varphi_{0}, \varphi_{1}, \ldots\right\}$ is an infinite dimensional vector consisting of two-way infinite sequence; then all of $\sum_{k=-\infty}^{+\infty}\left|\varphi_{k}\right|^{p}$ which satisfy $\sum_{k=-\infty}^{+\infty}\left|\varphi_{k}\right|^{p}<$ $+\infty$ constitute a linear space; we denote it as $\varphi \in \bar{l}^{P}$.

Remark 2. From Definition 1, we know that if $\varphi \in l^{p}$, then $c \varphi \in l^{p}$ for any constant $c$. Moreover, if $\left\{\varphi_{k}\right\} \in l^{p},\left\{\psi_{k}\right\} \in l^{q}$, then $\left\{\varphi_{k} \psi_{k}\right\} \in l^{1}$, where $p^{-1}+q^{-1}=1(p>1, q>1)$. In this paper we mainly consider the case $p=2$.

Definition 3. Let $\Phi(\theta), \Psi(\theta)$ be periodic functions with period $2 \pi$, and $\Phi(\theta), \Psi(\theta) \in L^{2}[-\pi, \pi]$; then

$$
(\Phi * \Psi)(\theta)=\frac{1}{2 \pi} \int_{-\pi}^{\pi} \Phi(\theta-\nu) \Psi(\nu) d \nu, \quad \theta \in[-\pi, \pi]
$$

is called the convolution of $\Phi(\theta)$ and $\Psi(\theta)$.

Remark 4. With regard to the convolution $(\Phi * \Psi)(\theta)$, we can extend the value of $\theta$ from $[-\pi, \pi]$ to $\mathbb{R}$ by Definition 3 . 
Definition 5. Let $\varphi=\left\{\varphi_{k}\right\}_{k=-\infty}^{+\infty} \in l^{2}$; the transforms $V$ and $V^{-1}$ defined by

$$
\begin{gathered}
V \varphi=\sum_{k=-\infty}^{+\infty} \varphi_{k} e^{i k \theta}=\Phi(\theta), \\
V^{-1}[\Phi(\theta)]=\frac{1}{2 \pi} \int_{-\pi}^{\pi} \Phi(\theta) e^{-i k \theta} d \theta=\varphi_{k}, \\
k=0, \pm 1, \pm 2, \ldots,
\end{gathered}
$$

are called the discrete Fourier transform and the inverse transform, respectively. For simplification, in (2), we denote them as $V \varphi=\Phi(\theta)$ and $V^{-1}[\Phi(\theta)]=\varphi_{k}$, respectively.

Definition 6. Let $\varphi=\left\{\varphi_{k}\right\}_{k=-\infty}^{+\infty}$ be a number sequence and $M$ and $\eta$ be positive constants with $0<\eta \leq 1$. If $\left|k^{1+\eta} \varphi_{k}\right| \leq M$ holds for any integer $k$, we say that $\varphi=\left\{\varphi_{k}\right\}$ belongs to class $\{1\}$.

Definition 7. If $\varphi=\left\{\varphi_{k}\right\}_{k=-\infty}^{+\infty} \in\{1\}$, then we say $\Phi(\theta) \in\{\widetilde{0}\}$.

The following lemma plays an important role, and it is proposed firstly in this paper.

Lemma 8. Let $\Phi(\theta) \in L^{2}[-\pi, \pi], S \Phi(\theta)=(1 /$ $\pi) \int_{-\pi}^{\pi} \Phi(\nu) \csc (\nu-\theta) d v$

$$
\widetilde{S} \Phi(\theta)=\frac{1}{\pi} \int_{-\pi}^{\pi} \Phi(\nu) \csc (\nu+\theta) d \nu .
$$

Then

$$
\begin{aligned}
& \text { (1) } V^{-1}(S \Phi(\theta))=2 \varphi_{k} i \operatorname{sgn} k \rho_{k}, \quad V^{-1}(\widetilde{S} \Phi(\theta))= \\
& -2 \varphi_{k} i \operatorname{sgn} k \rho_{k} ; \\
& \text { (2) } S \Phi(\theta) \in L^{2}[-\pi, \pi], V^{-1}(S \Phi(\theta)) \in l^{2}[-\pi, \pi],
\end{aligned}
$$

where $S \Phi(\theta), \widetilde{S} \Phi(\theta)$ are singular integrals with cosecant kernels and

$$
\rho_{k}=\left\{\begin{array}{ll}
1, & k=2 n+1 ; \\
0, & k=2 n,
\end{array} \quad n \in \mathbb{Z} .\right.
$$

Proof. (1) Since

$$
\begin{aligned}
S \Phi(\theta) & =\frac{1}{\pi} \int_{-\pi}^{\pi} \Phi(\nu) \csc (\nu-\theta) d \nu \\
& =\frac{1}{\pi} \int_{-\pi}^{\pi} \Phi(\nu) \frac{2 i}{e^{i(\nu-\theta)}-e^{-i(v-\theta)}} d \nu \\
& =\frac{2 i}{\pi} \int_{-\pi}^{\pi} \Phi(\nu) \frac{e^{i(\theta+\nu)}}{e^{2 i v}-e^{2 i \theta}},
\end{aligned}
$$

by Definition 5, we have

$$
\begin{aligned}
& V^{-1}(S \Phi(\theta)) \\
& \quad=i \cdot \frac{1}{\pi^{2}} \int_{-\pi}^{\pi} \Phi(\nu) e^{i \nu}\left[\int_{-\pi}^{\pi} \frac{e^{-i(k-1) \theta}}{e^{2 i \nu}-e^{2 i \theta}} d \theta\right] d \nu .
\end{aligned}
$$

It follows from the extended Residue theorem (see [9]) that

$$
\int_{-\pi}^{\pi} \frac{e^{-i(k-1) \theta}}{e^{2 i v}-e^{2 i \theta}} d \theta=\frac{\pi}{e^{i(k+1) \nu}} \rho_{k} \operatorname{sgn} k .
$$

This implies

$$
\begin{aligned}
V^{-1}(S \Phi(\theta)) & =i \frac{1}{\pi^{2}} \pi \rho_{k} \operatorname{sgn} k \int_{-\pi}^{\pi} \frac{1}{e^{i(k+1) \nu}} \Phi(\nu) e^{i v} d \nu \\
& =2 i \varphi_{k} \rho_{k} \operatorname{sgn} k .
\end{aligned}
$$

The other equality can be proved similarly.

(2) By using Riesz's theory (see [9]), we have $\|S \Phi(\theta)\|_{2} \leq$ $C\|\Phi(\theta)\|_{2}$, where $C$ is a constant. Therefore, it follows from $\Phi(\theta) \in L^{2}[-\pi, \pi]$ that $S \Phi(\theta) \in L^{2}[-\pi, \pi]$.

It is also obvious for the second conclusion in (2).

Lemma 9. If $\varphi=\left\{\varphi_{k}\right\}_{k=-\infty}^{+\infty}$ belongs to class $\{1\}$, then $\Phi(\theta)=$ $V \varphi \in H$, where $H$ is the class of Hölder continuous function.

Proof. By Definition 6, we know that $\sum_{k=-\infty, k \neq 0}^{+\infty}\left(M /|k|^{1+\eta}\right)$ converges; therefore, $\sum_{k=-\infty}^{\infty} \varphi_{k}$ is convergent absolutely. For any two points $\theta_{1}, \theta_{2} \in[-\pi, \pi]$, we have

$$
\begin{aligned}
\left|\Phi\left(\theta_{1}\right)-\Phi\left(\theta_{2}\right)\right| & =\left|\sum_{k=-\infty}^{\infty} \varphi_{k}\left(e^{i k \theta_{1}}-e^{i k \theta_{2}}\right)\right| \\
& \leq\left|\sum_{k=-\infty}^{\infty}\right| \varphi_{k}\left|\left(e^{i k \theta_{1}}-e^{i k \theta_{2}}\right)\right| \\
& \leq \widetilde{M}\left|\theta_{1}-\theta_{2}\right|,
\end{aligned}
$$

where $\widetilde{M}=\sum_{k=-\infty}^{\infty}\left|\varphi_{k}\right|$; thus $\Phi(\theta) \in H$.

Lemma 10. Let $\Phi(\theta)=\sum_{k=-\infty}^{\infty} \varphi_{k} e^{i k \theta}$ and $\varphi \in l^{1}$; then $\Phi(\theta) \epsilon$ $L^{2}[-\pi, \pi]$ if and only if $\varphi \in l^{2}$.

Proof. Since $\Phi(\theta) \in L^{2}[-\pi, \pi]$ and $\varphi \in l^{1}$, then $\sum_{k=-\infty}^{\infty} \varphi_{k}$ is convergent absolutely and uniformly. It is easy to see that

$$
\begin{aligned}
\int_{-\pi}^{\pi}|\Phi(\theta)|^{2} d \theta & =\int_{-\pi}^{\pi} \Phi(\theta) \overline{\Phi(\theta)} d \theta \\
& =\int_{-\pi}^{\pi} \sum \varphi_{k} e^{i k \theta} \sum \overline{\varphi_{j}} e^{-i j \theta} d \theta \\
& =\int_{-\pi}^{\pi} \sum_{k, j=-\infty}^{\infty} \varphi_{k} \overline{\varphi_{j}} e^{i(k-j) \theta} d \theta \\
& =2 \pi \sum_{k=-\infty}^{\infty}\left|\varphi_{k}\right|^{2} .
\end{aligned}
$$

The proof of Lemma 10 is complete.

Lemma 11 is an obvious fact.

Lemma 11 (convolution theorem). If $\Phi(\theta)$ and $\Psi(\theta) \quad \epsilon$ $L^{2}[-\pi, \pi]$, then $\Phi * \Psi(\theta) \in L^{2}[-\pi, \pi]$ and $V^{-1}[\Phi * \Psi(\theta)]=$ $\varphi_{k} \psi_{k}$, where $\psi_{k}=V^{-1}[\Psi(\theta)]$. 


\section{Problem Presentation and Methods of Solution}

Consider the following singular integral equation of convolution type with periodicity and cosecant kernels:

$$
\begin{aligned}
\xi_{1}(\theta) & {\left[a \Phi(\theta)+b S \Phi(\theta)+K_{1} * \Phi(\theta)+M_{1} * S \Phi(\theta)\right.} \\
- & c G(\theta)]+\xi_{2}(\theta)\left[a \Phi(\theta)+b S \Phi(\theta)+K_{2} * \Phi(\theta)\right. \\
+ & \left.M_{2} * S \Phi(\theta)-c G(\theta)\right]=0, \quad \theta \in \mathbb{R}
\end{aligned}
$$

where

$$
\begin{gathered}
\xi_{1}(\theta)=c_{1} \cos \frac{m \theta}{2}+d_{1} \sin \frac{m \theta}{2}, \\
\xi_{2}(\theta)=a_{1} \cos \frac{m \theta}{2}+b_{1} \sin \frac{m \theta}{2}, \\
M * S \Phi(\theta)=\frac{1}{2 \pi} \int_{-\pi}^{\pi} M(\theta-\nu) S \Phi(\nu) d \nu .
\end{gathered}
$$

$S \Phi(\theta)$ is as in Lemma 8. $a, b, c, a_{1}, b_{1}, c_{1}, d_{1}$ are real constants with $a^{2}+b^{2} \neq 0, a_{1} d_{1}-b_{1} c_{1} \neq 0$. The functions $\xi_{j}(\theta)(j=1,2)$ are periodic coefficients; $m$ is a positive integer. $K_{j}(\theta), M_{j}(\theta)$, $\Phi(\theta), S \Phi(\theta)$, and $G(\theta)$ are periodic functions with period $2 \pi$. Once $\Phi(\theta)$ is obtained in $[-\pi, \pi]$, we can make the periodic extension of $\Phi(\theta)$ with period $2 \pi$ and then obtain the solution $\Phi_{*}(\theta)$ in $\mathbb{R}$. Therefore, in the following discussion we are restricted to $\theta \in[-\pi, \pi]$.

Equation (11) is one important class of equations in the theory of integral equations, and it has important applications in physics, air dynamics, and electronic optical (e.g., see [10-14]). Hence, the study of (11) is meaningful not only in application but also in the method of solution.

Since $e^{i m \theta}=\cos m \theta+i \sin m \theta$, we write (11) as

$$
\begin{aligned}
& A^{+} \Phi(\theta)+B^{+} S \Phi(\theta)+\beta^{+} K_{1} * \Phi(\theta)+\alpha^{+} K_{2} * \Phi(\theta) \\
& \quad+\beta^{+} M_{1} * \Phi(\theta)+\alpha^{+} M_{2} * S \Phi(\theta)-F^{+} G(\theta) \\
& +e^{i m \theta}\left[A^{-} \Phi(\theta)+B^{-} S \Phi(\theta)+\beta^{-} K_{1} * \Phi(\theta)\right. \\
& +\alpha^{-} K_{2} * \Phi(\theta)+\beta^{-} M_{1} * \Phi(\theta)+\alpha^{-} M_{2} * S \Phi(\theta) \\
& \left.\quad-F^{-} G(\theta)\right]=0, \quad-\pi \leq \theta \leq \pi,
\end{aligned}
$$

where

$$
\begin{aligned}
& \alpha^{ \pm}=a_{1} \pm i b_{1}, \\
& \beta^{ \pm}=c_{1} \pm i d_{1}, \\
& A^{ \pm}=a\left[\left(a_{1}+c_{1}\right) \pm i\left(b_{1}+d_{1}\right)\right], \\
& B^{ \pm}=b\left[\left(a_{1}+c_{1}\right) \pm i\left(b_{1}+d_{1}\right)\right], \\
& F^{ \pm}=c\left[\left(a_{1}+c_{1}\right) \pm i\left(b_{1}+d_{1}\right)\right],
\end{aligned}
$$

and $\xi_{j}(\theta)(j=1,2)$ can be rewritten as

$$
\begin{aligned}
& \xi_{1}(\theta)=\frac{1}{2}\left(\beta^{-} e^{i m \theta / 2}+\beta^{+} e^{-i m \theta / 2}\right), \\
& \xi_{2}(\theta)=\frac{1}{2}\left(\alpha^{-} e^{i m \theta / 2}+\alpha^{+} e^{-i m \theta / 2}\right) .
\end{aligned}
$$

Let

$$
\begin{aligned}
& A^{-} \Phi(\theta)+B^{-} S \Phi(\theta)+\beta^{-} K_{1} * \Phi(\theta)+\alpha^{-} K_{2} * \Phi(\theta) \\
& \quad+\beta^{-} M_{1} * S \Phi(\theta)+\alpha^{-} M_{2} * S \Phi(\theta)-F^{-} G(\theta) \\
& =T(\theta),
\end{aligned}
$$

then,

$$
\begin{aligned}
A^{+} \Phi(\theta)+B^{+} S \Phi(\theta)+\beta^{+} K_{1} * \Phi(\theta)+\alpha^{+} K_{2} * \Phi(\theta) \\
\quad+\beta^{+} M_{1} * S \Phi(\theta)+\alpha^{+} M_{2} * S \Phi(\theta)-F^{+} G(\theta) \\
=-e^{i m \theta} T(\theta) ;
\end{aligned}
$$

Applying $V^{-1}$ to both sides of (16) and (17), respectively, by Lemma 8 we have

$$
\begin{aligned}
\tau_{k} & =\left(A^{-}+2 B^{-} i \operatorname{sgn} k \rho_{k}+\beta^{-} B_{k}^{(1)}+\alpha^{-} B_{k}^{(2)}\right. \\
& \left.+2 \beta^{-} C_{k}^{(1)} i \operatorname{sgn} k \rho_{k}+2 \alpha^{-} C_{k}^{(2)} i \operatorname{sgn} k \rho_{k}\right) \varphi_{k}-F^{-} g_{k} ; \\
- & \tau_{k-m}=\left(A^{+}+2 B^{+} i \operatorname{sgn} k \rho_{k}+\beta^{+} B_{k}^{(1)}+\alpha^{+} B_{k}^{(2)}\right. \\
& \left.+2 \beta^{+} C_{k}^{(1)} i \operatorname{sgn} k \rho_{k}+2 \alpha^{+} C_{k}^{(2)} i \operatorname{sgn} k \rho_{k}\right) \varphi_{k}-F^{+} g_{k},
\end{aligned}
$$

where

$$
\begin{aligned}
\tau_{k} & =V^{-1} T, \\
\varphi_{k} & =V^{-1} \Phi, \\
B_{k}^{(j)} & =V^{-1} K_{j}, \\
C_{k}^{(j)} & =V^{-1} M_{j} \\
& \quad(j=1,2), \\
g_{k} & =V^{-1} G .
\end{aligned}
$$

Denote

$$
\begin{aligned}
\pi_{k}^{ \pm}= & A^{ \pm}+2 B^{ \pm} i \operatorname{sgn} k \rho_{k}+\beta^{ \pm} B_{k}^{(1)}+\alpha^{ \pm} B_{k}^{(2)} \\
& +2 \beta^{ \pm} C_{k}^{(1)} i \operatorname{sgn} k \rho_{k}+2 \alpha^{ \pm} C_{k}^{(2)} i \operatorname{sgn} k \rho_{k} .
\end{aligned}
$$

Then (18) can be reduced as

$$
\begin{aligned}
& \pi_{k}^{-} \varphi_{k}-F^{-} g_{k}=\tau_{k} ; \\
& \pi_{k}^{+} \varphi_{k}-F^{+} g_{k}=-\tau_{k-m} .
\end{aligned}
$$

Since $\left\{B_{k}^{(j)}\right\}_{k=-\infty}^{\infty} \in l^{2},\left\{C_{k}^{(j)}\right\}_{k=-\infty}^{\infty} \in l^{2}(j=1,2)$, then $B_{k}^{(j)} \rightarrow$ $0, C_{k}^{(j)} \rightarrow 0$, and $\pi_{k}^{ \pm} \rightarrow A^{ \pm} \pm 2 i B^{ \pm} \neq 0$ or $\pi_{k}^{ \pm} \rightarrow A^{ \pm} \neq 0$ when 
$|k| \rightarrow \infty$. Therefore there exists $k_{0}\left(|k|>k_{0}\right)$ such that $\left|\pi_{k}^{ \pm}\right|>$ $\mu$ when $|k|>k_{0}$ for certain positive constant $\mu(0<\mu<1)$.

Consider first the case $|k| \leq k_{0}$.

(1) When $\pi_{k}^{ \pm}=0$ for $k=n_{1}, n_{2}, \ldots, n_{p_{1}}\left(-k_{0} \leq n_{j} \leq\right.$ $\left.k_{0}, 1 \leq j \leq p_{1}\right)$, by (21) we know that

$$
\begin{aligned}
\tau_{n_{j}} & =-F^{-} g_{n_{j}}, \\
\tau_{n_{j}-m} & =F^{+} g_{n_{j}}
\end{aligned}
$$

must be fulfilled. Now $\varphi_{n_{j}}\left(1 \leq j \leq p_{1}\right)$ take arbitrary constants.

(2) When $\pi_{k}^{+}=0, \pi_{k}^{-} \neq 0$ for $k=n_{1}^{\prime}, n_{2}^{\prime}, \ldots, n_{p_{2}}^{\prime}\left(-k_{0} \leq\right.$ $\left.n_{j}^{\prime} \leq k_{0}, 1 \leq j \leq p_{2}\right)$, by (21) we have

$$
\varphi_{n_{j}^{\prime}}=\frac{F^{-} g_{n_{j}^{\prime}}+\tau_{n_{j}^{\prime}}}{\pi_{n_{j}^{\prime}}^{-}},
$$

where

$$
\tau_{n_{j}^{\prime}}=F^{+} g_{n_{j}^{\prime}+m}
$$

(3) When $\pi_{k}^{+} \neq 0, \pi_{k}^{-}=0$ for $k=n_{1}^{\prime \prime}, n_{2}^{\prime \prime}, \ldots, n_{p_{3}}^{\prime \prime}\left(-k_{0} \leq\right.$ $\left.n_{j}^{\prime \prime} \leq k_{0}, 1 \leq j \leq p_{3}\right)$, by (21) we get

$$
\varphi_{n_{j}^{\prime \prime}}=\frac{F^{+} g_{n_{j}^{\prime \prime}}-\tau_{n_{j}^{\prime \prime}-m}}{\pi_{n_{j}^{\prime \prime}}^{+}}
$$

where

$$
\tau_{n_{j}^{\prime \prime}}=-F^{-} g_{n_{j}^{\prime \prime}}
$$

(4) When $\pi_{k}^{ \pm} \neq 0\left(-k_{0} \leq k \leq k_{0}\right), \varphi_{k}$ are given by (21) and this situation is similar to the situation $|k|>k_{0}$.

Next we consider the case $|k|>k_{0}$. Since $\left|\pi_{k}^{ \pm}\right|>\mu$ for $|k|>k_{0}$, then $\pi_{k}^{ \pm} \neq 0$. By (21) we obtain

$$
\varphi_{k}=\frac{1}{\pi_{k}^{-}}\left(\tau_{k}+F^{-} g_{k}\right)=\frac{1}{\pi_{k}^{+}}\left(-\tau_{k-m}+F^{+} g_{k}\right) ;
$$

thus

$$
\tau_{k}+R_{k} \tau_{k-m}=H_{k} g_{k}
$$

where

$$
\begin{aligned}
R_{k} & =\frac{\pi_{k}^{-}}{\pi_{k}^{+}} \\
H_{k} & =\frac{2 i\left(b_{1} c_{1}-a_{1} d_{1}\right)}{\pi_{k}^{+}}\left[\left(B_{k}^{(2)}-B_{k}^{(1)}\right)\right. \\
& \left.+2\left(C_{k}^{(2)}-C_{k}^{(1)}\right) i \operatorname{sgn} k \rho_{k}\right] .
\end{aligned}
$$

Assume that

$$
\begin{aligned}
\delta & =\frac{B^{-}}{B^{+}}, \\
R_{k} & =\delta\left(1+Y_{k}\right),
\end{aligned}
$$

where

$$
\begin{aligned}
Y_{k}= & \frac{2 i b\left(b_{1} c_{1}-a_{1} d_{1}\right)}{B^{-}} \\
& \cdot \frac{B_{k}^{(1)}-B_{k}^{(2)}+2 i \operatorname{sgn} k \rho_{k}\left[C_{k}^{(1)}-C_{k}^{(2)}\right]}{\pi_{k}^{+}} .
\end{aligned}
$$

Therefore, (28) can be written as the following discrete jump problem:

$$
\tau_{k}+\delta\left(1+Y_{k}\right) \tau_{k-m}=H_{k} g_{k}
$$

Thus, we should only study (32) in place of (11). Since $\left\{H_{k}\right\} \in$ $l^{2},\left\{g_{k}\right\} \in l^{2}$, then $\left\{H_{k} g_{k}\right\} \in l^{2},\left\{\tau_{k}\right\} \in l^{2}$. Once $\tau_{k}$ is obtained, then $\varphi_{k}$ can be given by (21). To solve (32), we first need to analyze the structure of $1+Y_{k}$. Assume that $1+T_{k}$ can be factorized; we can choose $r_{k}$ such that

$$
1+Y_{k}=\frac{r_{k}}{r_{k-m}}
$$

where $\mu<\left|r_{k}\right|<\mu^{-1} ; \mu$ is as the above. Provided that $\mu$ takes sufficiently small value, we can choose $r_{k}$ satisfying the above requirement (33). We now give an expression of $r_{k}$, taking logarithms for both sides of (33) and denoting

$$
\begin{aligned}
& w_{k}=\ln r_{k} \\
& u_{k}=\ln \left(1+Y_{k}\right) ;
\end{aligned}
$$

then, by (33) and (34) we obtain

$$
w_{k}-w_{k-m}=u_{k}
$$

Note that we have taken a continuous branch of $\ln \left(1+Y_{k}\right)$ such that $\ln \left(1+Y_{\mathrm{k}}\right) \in l^{2}$. Applying Fourier transform to both sides of (35), we can obtain

$$
\left(1-e^{i m \theta}\right) W(\theta)=U(\theta)
$$

where $W(\theta)=V w, U(\theta)=V u, w=\left\{w_{k}\right\}_{k=-\infty}^{+\infty}$, and $u=$ $\left\{u_{k}\right\}_{k=-\infty}^{+\infty}$.

From the above discussions, the following conditions of solvability $U\left(\theta_{j}\right)=0$ follow and that is,

$$
\sum\left(\ln \frac{1}{\delta} R_{k}\right) e^{i k \theta_{j}}=0, \quad\left\{\ln \frac{1}{\delta} R_{k}\right\} \in l^{2},
$$

where $\theta_{j}=(2 j / m) \pi(j=0, \pm 1, \ldots, \pm[m / 2] ;[m / 2]$ is the integer part of $m / 2)$. Therefore,

$$
W(\theta)=\frac{U(\theta)}{1-e^{i m \theta}} \quad\left(\theta \neq \theta_{j},|j| \leq 2\left[\frac{m}{2}\right]+1\right) .
$$

From (38), we obtain $w_{k}=V^{-1} W(\theta)$ and thus $r_{k}=\exp w_{k}$. Note (33) and denote

$$
\begin{aligned}
e_{k} & =\frac{\tau_{k}}{r_{k}}, \\
\psi_{k} & =\frac{H_{k} g_{k}}{r_{k}} .
\end{aligned}
$$


Then (32) can be rewritten as

$$
e_{k}+\delta e_{k-m}=\psi_{k} .
$$

Since $\left\{H_{k} g_{k}\right\} \in l^{2},\left\{r_{k}\right\} \in l^{2}$, and $\mu<\left|r_{k}\right|<\mu^{-1}$, we have $\left\{e_{k}\right\} \in l^{2}$ and $\left\{\psi_{k}\right\} \in l^{2}$. By taking Fourier transform $V$ for both sides of (40), we have

$$
\left(1+\delta e^{i m \theta}\right) E(\theta)=\Psi(\theta)
$$

where $E(\theta)=V e, \Psi(\theta)=V \psi, e=\left\{e_{k}\right\}_{k=-\infty}^{+\infty}$, and $\psi=$ $\left\{\psi_{k}\right\}_{k=-\infty}^{+\infty}$.

Since $|\delta|=1$, we know that $1+\delta e^{i m \theta}$ has finite number of zero points $\theta_{1}^{\prime}, \theta_{2}^{\prime}, \ldots, \theta_{n}^{\prime}$ in $[-\pi, \pi]$.

Therefore the conditions of solvability $\Psi\left(\theta_{j}^{\prime}\right)=0$, that is,

$$
\sum \frac{H_{k} g_{k}}{r_{k}} e^{i k \theta_{j}^{\prime}}=0, \quad\left\{\frac{H_{k} g_{k}}{r_{k}}\right\} \in l^{2}(j=1,2, \ldots, n),
$$

must be augmented. In view of (41) we get

$$
E(\theta)=\frac{\Psi(\theta)}{1+\delta e^{i m \theta}} \quad\left(\theta \neq \theta_{j}^{\prime}, j=1,2, \ldots, n\right) .
$$

If (37) and (42) are satisfied, then by (43) we obtain

$$
e_{k}=V^{-1} E(\theta) \quad(k=0, \pm 1, \ldots) .
$$

Thus $\tau_{k}(k=0, \pm 1, \ldots)$ can be also obtained by (39).

Now we state our main result.

Theorem 12. Under (37) and (42), (11) has a solution. When $\pi_{k}^{ \pm} \neq 0(k=0, \pm 1, \ldots), \varphi_{k}$ is given by $(21)$. When $\pi_{n_{j}}^{ \pm}=0(j=$ $\left.1,2, \ldots, p_{1}\right), \varphi_{n_{j}}$ take arbitrary constants. When $\pi_{n_{j}^{\prime}}^{+}=0$, $\pi_{n_{j}^{\prime}}^{-} \neq 0\left(j=1,2, \ldots, p_{2}\right), \varphi_{n_{j}^{\prime}}, \tau_{n_{j}^{\prime}}$ are given by (23) and (24), respectively. When $\pi_{n_{j}^{\prime \prime}}^{+} \neq 0, \pi_{n_{j}^{\prime \prime}}^{-}=0\left(j=1,2, \ldots, p_{3}\right), \varphi_{n_{j}^{\prime \prime}}, \tau_{n_{j}^{\prime \prime}}$ are given by (25) and (26), respectively. But in (23) and (25), $\tau_{n_{j}^{\prime}}$ and $\tau_{n_{j}^{\prime \prime}}$ are constants determined by (24) and (26), respectively. When $k \neq n_{j}, n_{j}^{\prime}, n_{j}^{\prime \prime}, \varphi_{k}$ is given by (21). Therefore, the solution of (11) is of the form

$$
\Phi(\theta)=V \varphi=\sum_{k=-\infty}^{\infty} \varphi_{k} e^{i k \theta}
$$

and then $\Phi(\theta) \in L^{2}[-\pi, \pi]$.

Proof. From the above discussion, we only need to prove that the function $\Phi(\theta)$ obtained by (45) belongs to $L^{2}[-\pi, \pi]$. Obviously, (11), (13), and (21) are equivalent to each other. Since $\left\{g_{k}\right\},\left\{\tau_{k}\right\} \in l^{2}$, then $\left\{\pi_{k}^{ \pm}\right\} \in l^{2}$ and $\pi_{k}^{ \pm} \neq 0$. It follows from (27) that $\left\{\varphi_{k}\right\} \in l^{2}$ is a bounded sequence, and $\sum_{k=-\infty}^{\infty} \varphi_{k} e^{i k \theta}$ is convergent. Thus, by Lemma 10, (11) has a unique solution $\Phi(\theta)=V \varphi$ in class $L^{2}[-\pi, \pi]$.

According to Theorem 12, Definition 5, and Lemma 9, we obtain the following.
Theorem 13. In (11), if $K_{j}(\theta), M_{j}(\theta)(j=1,2), G(\theta) \in\{\widetilde{0}\}$, then (11) is solvable in class $\{\widetilde{0}\}$ and all conditions of solvability and its solutions are similar to those in Theorem 12.

In order to illustrate that (11) has an explicit solution, we present an example and satisfy the above conditions (37) and (42). For example, suppose that

$$
\begin{aligned}
a & =b=c=1, \\
b_{1} & =c_{1}=0, \\
a_{1} & =d_{1} \neq 0, \\
K_{1} & =K_{2}, \\
M_{1} & =M_{2} ;
\end{aligned}
$$

then $\xi_{1}(\theta)=a_{1} \sin (m / 2) \theta, \xi_{2}(\theta)=a_{1} \cos (m / 2) \theta$, and (11) can be transformed into

$$
\Phi+S \Phi+K_{1} * \Phi+M_{1} * S \Phi=G
$$

it is easy to prove that (47) satisfies conditions (37) and (42), and the solution of (47) was obtained in the literature [1]. Therefore, we can conclude that a solution set of (11) is not empty.

Remark 14. If $a_{1} d_{1}-b_{1} c_{1}=0, \xi_{2}(\theta) / \xi_{1}(\theta)$ is a constant. This case is simple, and we do not discuss it here.

\section{Results and Discussion}

In this paper, we first proposed one class of singular integral equations of convolution type with cosecant kernels and periodic coefficients. By applying discrete Fourier transform and its properties, such equation can be transformed into a discrete jump problem depending on some parameter. Here, our method is different from the ones of the classical boundary value problem, and it is novel and simple. The exact solution, denoted by series, of (11) and the conditions of solvability are obtained in $L^{2}[-\pi, \pi]$. We remark that our approach is also effective in other classes of equations, such as the equations of dual type with periodicity and cosecant kernel and Wiener-Hopf type equations. Thus, this paper generalizes the classical theory of boundary value problems and singular integral equations.

In this paper, we solved (11) in $L^{2}[-\pi, \pi]$. Indeed, this class of equations can be also solved in Clifford analysis, which is similar to that in [15-18]. Further discussion is omitted here.

\section{Conclusions}

Equation (11) has important applications in practical problems, such as elastic mechanics, heat conduction, and electrostatics. Many problems, such as piezoelectric material, voltage magnetic materials, and functional gradient materials, can often attribute the problem to finding their solutions to (11). For the study of such equations, the present result is still rare due to lack of effective approaches. Our approach of solving 
the equations is novel, different from the ones in classical cases, and it is converted by using discrete Fourier transform into a discrete boundary value problem depending on some parameter; here we call it "a discrete jump problem." The exact solutions of (11) and the conditions of solvability are obtained in class $L^{2}[-\pi, \pi]$.

\section{Conflicts of Interest}

The author declares no conflicts of interest.

\section{Acknowledgments}

This work was supported by the Science and Technology Plan Project of Qufu Normal University (xkj 201606) and the author gratefully acknowledges this support.

\section{References}

[1] N. I. Musknelishvilli, Singular Integral Equations, Nauka: Moscow Press, 2002.

[2] J. K. Lu, Boundary Value Problems for Analytic Functions, World Scientific Publishing, Singapore, 1993.

[3] P. Li, "One class of generalized boundary value problem for analytic functions," Boundary Value Problems, vol. 40, 2015.

[4] T. Nakazi and T. Yamamoto, "Normal Singular Integral Operators with Cauchy Kernel on L 2," Integral Equations and Operator Theory, vol. 78, no. 2, pp. 233-248, 2014.

[5] P. Wójcik, M. A. Sheshko, and S. M. Sheshko, "Application of Faber polynomials to the approximate solution of singular integral equations with the Cauchy kernel," Differential Equations, vol. 49, no. 2, pp. 198-209, 2013.

[6] Y. Jiang and Y. Xu, "Fast Fourier-Galerkin methods for solving singular boundary integral equations: Numerical integration and precondition," Journal of Computational and Applied Mathematics, vol. 234, no. 9, pp. 2792-2807, 2010.

[7] H. Du and J. Shen, "Reproducing kernel method of solving singular integral equation with cosecant kernel," Journal of Mathematical Analysis and Applications, vol. 348, no. 1, pp. 308314, 2008.

[8] P. Li and G. Ren, "Some classes of equations of discrete type with harmonic singular operator and convolution," Applied Mathematics and Computation, vol. 284, pp. 185-194, 2016.

[9] R. Abreu-Blaya, J. Bory-Reyes, F. Brackx, H. De Schepper, and F. Sommen, "Cauchy Integral Formulae in Quaternionic Hermitean Clifford Analysis," Complex Analysis and Operator Theory, vol. 6, no. 5, pp. 971-985, 2012.

[10] P. Li, "Two classes of linear equations of discrete convolution type with harmonic singular operators," Complex Variables and Elliptic Equations, vol. 61, no. 1, pp. 67-75, 2016.

[11] O. A. Arqub, "Approximate Solutions of DASs with Nonclassical Boundary Conditions using Novel Reproducing Kernel Algorithm," Fundamenta Informaticae, vol. 146, no. 3, pp. 231-254, 2016.

[12] O. Abu Arqub and B. Maayah, "Solutions of Bagley-Torvik and Painlevé equations of fractional order using iterative reproducing kernel algorithm with error estimates," Neural Computing and Applications, pp. 1-15, 2016.

[13] O. Abu Arqub, “The reproducing kernel algorithm for handling differential algebraic systems of ordinary differential equations,"
Mathematical Methods in the Applied Sciences, vol. 39, no. 15, pp. 4549-4562, 2016.

[14] D. Jinyuan, X. Na, and Z. Zhongxiang, "Boundary behavior of Cauchy-type integrals in Clifford analysis," Acta Mathematica Scientia, vol. 29, no. 1, pp. 210-224, 2009.

[15] G. Ren, U. Kähler, J. Shi, and C. Liu, "Hardy-Littlewood Inequalities for Fractional Derivatives of Invariant Harmonic Functions," Complex Analysis and Operator Theory, vol. 6, no. 2, pp. 373-396, 2012.

[16] M. C. De Bonis and C. Laurita, "Numerical solution of systems of Cauchy singular integral equations with constant coefficients," Applied Mathematics and Computation, vol. 219, no. 4, pp. 1391-1410, 2012.

[17] P. Li, "Generalized convolution-type singular integral equations," Applied Mathematics and Computation, vol. 311, pp. 314323, 2017.

[18] O. Abu Arqub, "Fitted reproducing kernel Hilbert space method for the solutions of some certain classes of time-fractional partial differential equations subject to initial and Neumann boundary conditions," Computers \& Mathematics with Applications, vol. 73, no. 6, pp. 1243-1261, 2017. 


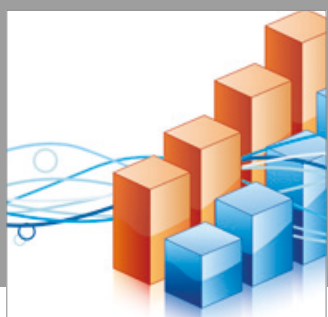

Advances in

Operations Research

vatersals

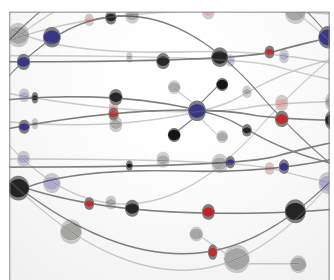

\section{The Scientific} World Journal
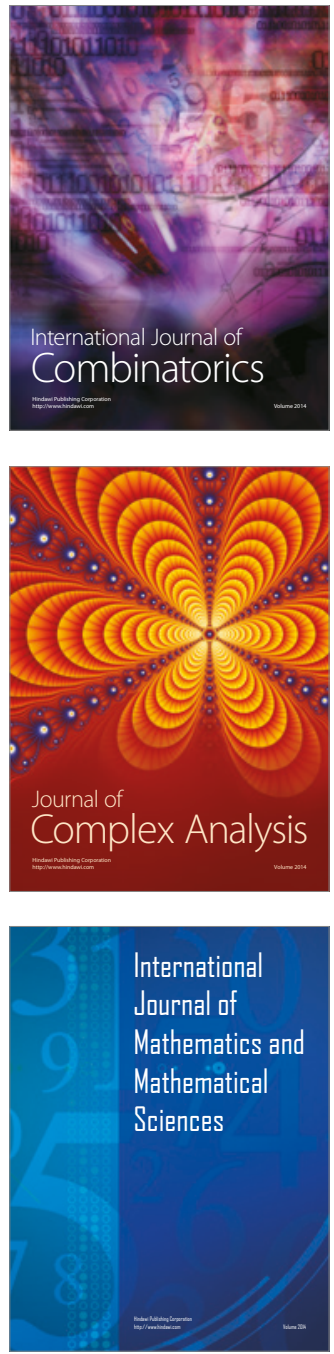
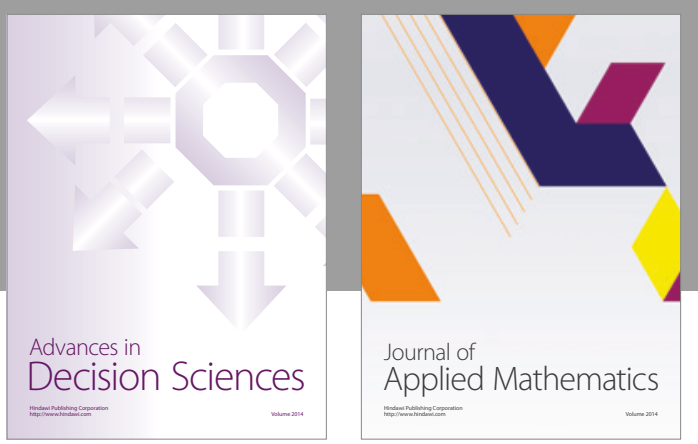

Algebra

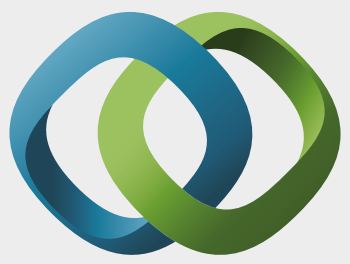

\section{Hindawi}

Submit your manuscripts at

https://www.hindawi.com
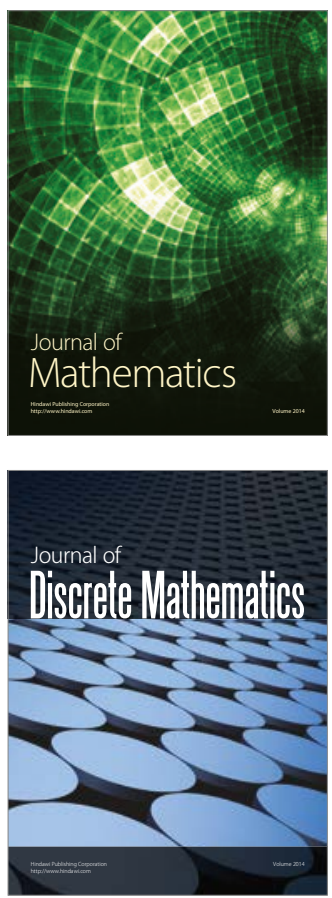

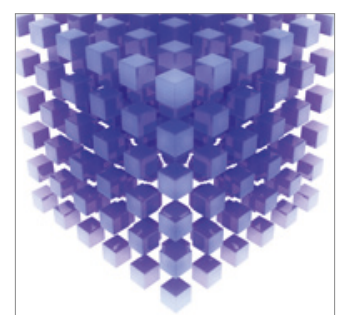

Mathematical Problems in Engineering
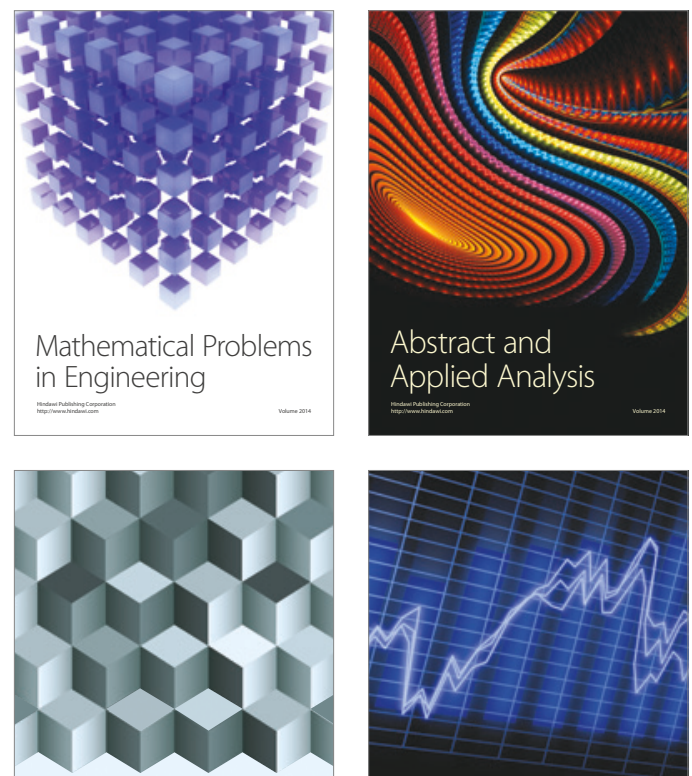

Journal of

Function Spaces

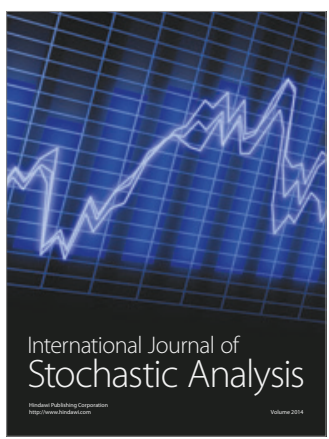

Probability and Statistics
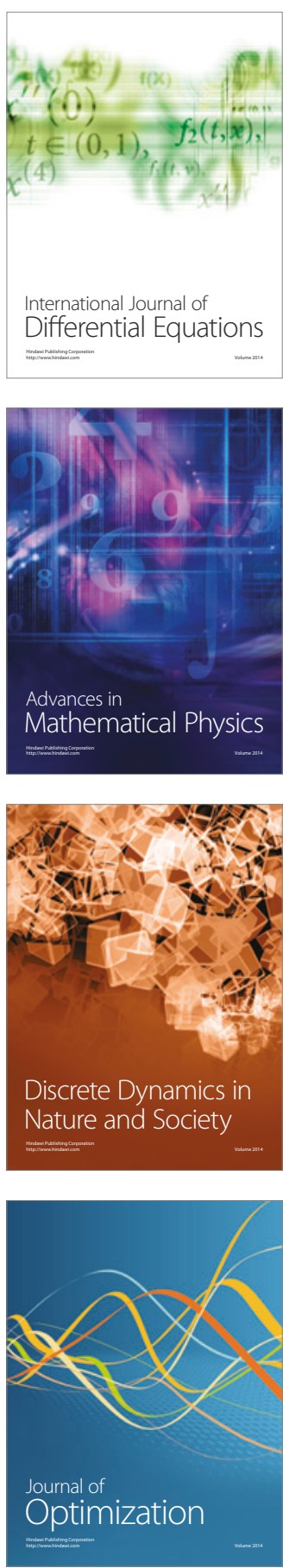